\title{
Protective capacities of cell surface-associated proteins of Streptococcus suis mutants deficient in divalent cation-uptake regulators
}

\begin{abstract}
Correspondence
Jordi Barbé

jordi.barbe@uab.cat
\end{abstract}

Received 28 November 2008

Revised 22 January 2009

Accepted 23 January 2009
Jesús Aranda, ${ }^{1}$ Maria Elena Garrido, ${ }^{1,2}$ Nahuel Fittipaldi, ${ }^{3}$ Pilar Cortés, ${ }^{1,4}$ Montserrat Llagostera, ${ }^{1,2}$ Marcelo Gottschalk ${ }^{3}$ and Jordi Barbé ${ }^{1,2}$

\author{
'Department de Genètica i Microbiologia, Universitat Autònoma de Barcelona (UAB), Bellaterra, \\ 08193 - Barcelona, Spain \\ ${ }^{2}$ Centre de Recerca en Sanitat Animal (CReSA), Bellaterra, 08193 - Barcelona, Spain \\ ${ }^{3}$ Groupe de Recherche sur les Maladies Infectieuses du Porc and Centre de Recherche en \\ Infectiologie Porcine, Faculté de Médecine Vétérinaire, Université de Montréal, St-Hyacinthe, OC \\ J2S 7C6, Canada \\ ${ }^{4}$ Servei d'Anàlisis i d'Aplicacions Microbiològiques, UAB, Bellaterra, 08193 - Barcelona, Spain
}

\begin{abstract}
Many cell surface-associated, divalent cation-regulated proteins are immunogenic, and some of them confer protection against the bacterial species from which they are derived. In this work, two Streptococcus suis divalent cation uptake regulator genes controlling zinc/manganese and iron uptake (adcR and fur, respectively) were inactivated in order to study the protective capacities of their cell surface-associated proteins. The results obtained showed overexpression of a set of immunogenic proteins (including members of the pneumococcal histidine triad family previously reported to confer protection against streptococcal pathogens) in $S$. suis adcR mutant cell surface extracts. Likewise, genes encoding zinc transporters, putative virulence factors and a ribosomal protein paralogue related to zinc starvation appeared to be derepressed in this mutant strain. Moreover, protection assays in mice showed that although neither adcR-nor fur-regulated cell surface-associated proteins were sufficient to confer protection in mice, the combination of both adcR- and fur-regulated cell surface-associated proteins is able to confer significant protection ( $50 \%, P=0.038)$ against a challenge to mice vaccinated with them.
\end{abstract}

\section{INTRODUCTION}

Streptococcus suis is an important pathogen that causes significant economic losses in the swine industry worldwide (Higgins et al., 1995). Infection caused by S. suis may vary from asymptomatic bacteraemia to fulminant systemic disease, with meningitis as the most outstanding feature (Higgins et al., 1995). It is also an important zoonotic agent for humans, who may acquire the infection through skin wounds upon contact with pigs and/or their by-products. Recently, during an outbreak in China, 215 human cases of S. suis infection were documented, and 39 of those patients died (Yu et al., 2006). More than 30 serotypes of S. suis, based on its capsular antigens, are currently known (Higgins et al., 1995). Serotype 2 is the most frequently isolated from diseased animals (Staats et al., 1997). Efforts to control

Abbreviations: ABC, ATP-binding cassette; EMSA, electrophoretic mobility shift assay.

Two supplementary tables, listing bacterial strains and plasmids and oligonucleotide primers used in this work, are available with the online version of this paper. infection are hampered by the lack of effective vaccines against S. suis. Furthermore, mechanisms involved in pathogenesis of this micro-organism are not yet completely understood (Gottschalk \& Segura, 2000).

Iron and zinc are essential components of many bacterial proteins either as structural or catalytic cofactors (Andrews et al., 2003; Babich \& Stotzky, 1978). The concentration of both elements in the free state in the body fluids of mammals is extremely low in order to prevent bacterial proliferation. On the other hand, metals at high concentrations are toxic to micro-organisms. Toxicity occurs through the displacement of essential metals from their native binding sites or through ligand interactions (Babich \& Stotzky, 1978; Chamnongpol et al., 2002). In addition, iron can be particularly toxic under aerobic conditions, interacting with either oxygen or oxygen-reduced species (Touati, 2000). Consequently, micro-organisms require homeostatic mechanisms to control intracellular metal levels.

Fur (ferric uptake regulator) is a transcriptional regulator of genes involved in iron uptake and storage in many 
bacterial species (Andrews et al., 2003). In Escherichia coli and Bacillus subtilis, and in species closely related to them, transcription of the zinc transporter genes is regulated by Zur (zinc uptake regulator), which belongs to the Fur protein family (Patzer \& Hantke, 2000). However, in Streptococcus species, zinc transporter genes are controlled by AdcR, a transcriptional regulatory factor of the MarR protein family (Loo et al., 2003).

Several cell surface-associated transporters involved in divalent cation uptake, including ATP-binding cassette $(\mathrm{ABC})$ transporters, have been shown to elicit a protective immune response (Garmory \& Titball, 2004; Tanabe et al., 2006). It has been shown that in S. suis, AdcR controls at least two operons related to $\mathrm{Zn}^{2+} / \mathrm{Mn}^{2+}$ uptake, encoding a complete ABC transporter (SsuiDRAFT 1237-1240) and a divalent cation-binding lipoprotein (SsuiDRAFT 0103) (Aranda et al., 2008). Interestingly, both cation-binding lipoproteins encoded by these operons (SsuiDRAFT 1237 and SsuiDRAFT 0103) are immunogenic when analysed on Western blots using immunologically reactive serum obtained from mice surviving after inoculation with the wild-type S. suis 89/1591 strain (Aranda et al., 2008). Furthermore, a high level of protection against $S$. suis challenge is obtained in mice when vaccinated with the product of the ssuiDRAFT 0103 ORF (Aranda et al., 2008).

Different strategies have been developed to produce vaccines against pathogenic bacteria, including overexpression on the bacterial cell surface of divalent cation-uptake transporters which are induced in the presence of chelating agents, or by the construction of strains deficient in cationuptake repressors (Garrido et al., 2008). In this context, the aim of this work has been to obtain knockout $S$. suis mutants for the regulator genes of either zinc/manganeseor iron-uptake systems to study their possible use as a protective tool against this pathogen.

\section{METHODS}

Bacterial strains, plasmids and growth conditions. Bacterial strains and plasmids used in this work are listed in Supplementary Table S1. E. coli cells were grown in Luria-Bertani (LB) medium (Miller, 1992). When necessary, ampicillin $\left(50 \mu \mathrm{g} \mathrm{ml}^{-1}\right)$, kanamycin $\left(50 \mu \mathrm{g} \mathrm{ml}^{-1}\right)$ or spectinomycin $\left(50 \mu \mathrm{g} \mathrm{ml}^{-1}\right)$ was added to $\mathrm{LB}$ medium. S. suis was grown in Todd-Hewitt medium (TH medium; Difco). When required, spectinomycin $\left(100 \mu \mathrm{g} \mathrm{ml}^{-1}\right)$ was added to TH medium. Unless otherwise indicated, all cultures were incubated at $37{ }^{\circ} \mathrm{C}$, with shaking for E. coli. DNA extractions, cloning, and other molecular techniques used were performed as described elsewhere (Sambrook \& Russell, 2001). All oligonucleotides (Roche) used in this work are listed in Supplementary Table S2.

Construction of S. suis mutants. The $a d c R$ and fur genes were inactivated by precise in-frame deletion. Briefly, upstream and downstream regions of both $a d c R$ and fur genes (including partial coding regions) were amplified by overlap-extension PCR using genomic DNA from S. suis P1/7 strain as a template. A PCR product of 892 bp with a deletion of $229 \mathrm{bp}$ was obtained for the adcR gene, and a PCR product of 848 bp with a deletion of $222 \mathrm{bp}$ was obtained for the fur gene. Both PCR fragments were cloned into the pCR4-
TOPO vector following the manufacturer's instructions and propagated in E. coli One Shot TOP 10 cells. Inserts were amplified afterwards by PCR using primers M13F and M13R (Supplementary Table S2), amplicons were digested with EcoRI and cloned into the EcoRI-digested thermosensitive suicide vector pSET4s, which carries the $s p c$ and repAts genes conferring spectinomycin resistance and thermosensitivity, respectively (Takamatsu et al., 2001b), giving rise to plasmids pSET4 $\Delta$ adcR and pSET4 $\Delta$ fur. Recombinant plasmids were propagated in E. coli ABLE-C cells, purified, and used to electroporate $S$. suis electrocompetent P1/7 and UA5000 strains, as described elsewhere (Takamatsu et al., 2001a). Cells were then plated on TH agar containing spectinomycin and incubated for $48 \mathrm{~h}$ at $28{ }^{\circ} \mathrm{C}$ to select for clones that had incorporated the plasmid. Several clones were then selected and purified by single-colony isolation on $\mathrm{TH}$ agar containing spectinomycin and incubated at $37^{\circ} \mathrm{C}$. Integration of the construct into the chromosome as a result of homologous recombination via a single crossover was verified by PCR in temperature- and spectinomycin-resistant colonies. These colonies were then passaged several times onto non-selective media at $28{ }^{\circ} \mathrm{C}$ and screened for loss of vector-mediated spectinomycin resistance. Candidate spectinomycin-sensitive, temperature-resistant clones were analysed by PCR, which showed the exchange of the wild-type allele for the genetic segment containing the desired deletion as a consequence of a homologous recombination through a doublecrossover event. Allelic replacement in candidate clones was further confirmed by sequencing of the region in the resulting mutants.

Preparation of $\mathbf{S}$. suis cell surface-associated proteins. Extraction of these proteins was performed as described elsewhere (Tavares \& Sellstedt, 2000), with some modifications. Briefly, cells from $200 \mathrm{ml}$ overnight culture were pelleted at $4{ }^{\circ} \mathrm{C}$ by centrifugation at $8000 \mathrm{~g}$ for $10 \mathrm{~min}$. The cells were washed with $62.5 \mathrm{mM}$ Tris/ $\mathrm{HCl}$ $(\mathrm{pH}$ 6.8) and the pellet was pipetted up and down for $5 \mathrm{~min}$ in $4 \mathrm{ml}$ of the same buffer supplemented with $0.1 \%(\mathrm{v} / \mathrm{v})$ Triton X-100 (Roche). The suspension was centrifuged at $8000 \mathrm{~g}$ for $10 \mathrm{~min}$ at $4{ }^{\circ} \mathrm{C}$. The supernatant was collected and centrifuged for $2 \mathrm{~h}$ at $51030 \mathrm{~g}$ at $4{ }^{\circ} \mathrm{C}$ in order to concentrate the cell surface-associated protein extract. Finally, the protein pellet was resuspended in $100 \mu \mathrm{l} 62.5 \mathrm{mM}$ Tris/HCl (pH 6.8), visualized by SDS-PAGE (Laemmli, 1970) and stored at $4{ }^{\circ} \mathrm{C}$. The SDS-PAGE gel was stained with Brilliant Blue $\mathrm{G}$ Colloidal (Sigma) in order to avoid any stain interference with the subsequent procedures. The identification of overexpressed proteins was done by the Servei de Proteòmica i Bioinformàtica (SepBio) of the Universitat Autònoma de Barcelona (UAB). Thus, electrophoretic bands were in-gel digested with trypsin and analysed by MALDI-TOF MS as previously reported (Abella et al., 2007).

RNA techniques. RNA was isolated as described elsewhere (Fontaine et al., 2004), with slight modifications. A $10 \mathrm{ml}$ sample of an S. suis culture in the mid-exponential growth phase $\left(\mathrm{OD}_{600} 0.6\right)$ was collected by centrifugation at $8000 \mathrm{~g}$ for $10 \mathrm{~min}$. Cells were then resuspended by vigorous vortexing at $37{ }^{\circ} \mathrm{C}$ for $10 \mathrm{~min}$ in $300 \mu \mathrm{l}$ TrisEDTA prelysis buffer containing $10 \mathrm{mg}$ lysozyme $\mathrm{ml}^{-1}$. Total RNA was extracted by using the RNeasy Mini kit (Qiagen) according to the manufacturer's instructions. DNA contamination was removed from the RNA during purification by treatment with RNase-free DNase (Qiagen), followed by digestion with DNase Turbo (Ambion). The concentration and integrity of RNA were determined by $A_{260}$ measurements and $1 \%$ agarose gel electrophoresis, respectively. RTPCR assays were done using a Titan One Tube RT-PCR system (Roche) following the manufacturer's instructions. Real-time RTPCR analysis of gene expression was performed for all genes as reported previously (Campoy et al., 2002), with specific internal oligonucleotides used for each gene (Supplementary Table S2). In all cases, the absence of DNA in RNA samples was tested by PCR without the addition of reverse transcriptase. 
Mobility shift assays. Electrophoretic mobility shift assays (EMSAs) were done as described elsewhere (Bsat \& Helmann, 1999) with slight modifications. DNA promoters were PCR-amplified from P1/7 S. suis genomic DNA using suitable oligonucleotides (Supplementary Table S2), and the purified PCR fragments were cloned in E. coli $\mathrm{DH} 5 \alpha$ cells via the pGEM-T vector. The presence of the desired promoter was confirmed by sequencing the plasmid DNA using the primers M13F and M13R. DNA probes were prepared by PCR amplification, using primers of which one was labelled with DIG at its $5^{\prime}$ end (Supplementary Table S2). DNA-protein reaction mixtures $(20 \mu \mathrm{l})$ containing $25 \mathrm{ng}$ DIG-DNA-labelled probe and either 0 or $10 \mu \mathrm{g}$ purified S. suis AdcR protein were incubated in EMSA buffer [ $20 \mathrm{mM}$ Tris/ $\mathrm{HCl}(\mathrm{pH} 8), 50 \mathrm{mM} \mathrm{KCl}, 5 \%$ (v/v) glycerol, $1 \mu \mathrm{g}$ bulk carrier salmon sperm DNA, $0.5 \mathrm{mM} \mathrm{DTT}$, and $0.1 \mathrm{mg} \mathrm{BSA} \mathrm{ml}^{-1}$ ] for $10 \mathrm{~min}$ at room temperature. DNA-protein complexes were visualized by separation on a $5 \%$ non-denaturing polyacrylamide gel $(40 \mathrm{mM}$ Tris/ acetate) at $150 \mathrm{~V}$ for $1.5 \mathrm{~h}$ and then transferred to a Biodine B nylon membrane (Pall Gelman Laboratory). DIG-DNA-labelled protein complexes were detected according to the manufacturer's protocol (Roche). AdcR protein was purified as previously described (Aranda et al., 2008).

Protection assays. Female BALB/cAnNHsd mice (3 weeks old) obtained from Harlan Iberica were used for these studies. All animals underwent a quarantine period of 1 week. A maximum of four animals per cage were housed under specific-pathogen-free conditions at $19-21{ }^{\circ} \mathrm{C}$ with an artificial $12 \mathrm{~h}$ light/ $12 \mathrm{~h}$ dark cycle; the relative humidity was 50-60\%. Antibiotic-free pelleted food and autoclaved water were provided ad libitum during the experiments. Animals were monitored daily for morbidity and mortality. All animal experiments were approved by the UAB Animal Ethics Committee. For protection assays, groups of eight female 4-week-old $\mathrm{BALB} / \mathrm{cAnNHsd}$ mice were given intraperitoneal injections $(0.1 \mathrm{ml})$ of $50 \mu \mathrm{g}$ cell surface-associated proteins adsorbed with Imject Alum adjuvant (aluminium hydroxide; Pierce) according to the manufacturer's protocol. One additional group of eight mice was given injections of $0.1 \mathrm{ml}$ solvent including Imject Alum adjuvant as a negative control. After 2 weeks, a second immunization was carried out, followed 3 weeks later by a challenge with an intraperitoneal injection of a $0.1 \mathrm{ml}$ suspension of TH broth supplemented with $10 \%$ inactivated bovine serum containing $10^{8}$ c.f.u. S. suis $89 / 1591$. The survival of the mice was subsequently monitored for 7 days. Serum for Western blot assays was obtained as previously reported (Aranda et al., 2008).

Statistical analysis. Fisher's exact test was used to analyse the statistical significance of the lethal challenge data. A statistical test with a $P$ value less than 0.05 was considered to be significant.

\section{RESULTS AND DISCUSSION}

\section{Analysis of the $S$. suis adcR regulon composition}

It has been predicted in silico and demonstrated in vitro that the TTAACNRGTTAA motif is the AdcR DNAbinding sequence (Aranda et al., 2008; Panina et al., 2003). A search in the complete sequenced genome of $S$. suis strain P1/7 (http://www.sanger.ac.uk/Projects/S_suis/) with the EditSeq program (DNASTAR) revealed four ORFs with a motif identical to the AdcR-binding sequence in their promoter regions.

Two of the ORFs are ssu1390 and ssu1352, whose predicted products are homologous, respectively, to internalin A (InlA) of Listeria monocytogenes [which allows this bacterium to invade cells (Wollert et al., 2007)] and to the 30 S ribosomal protein S14, which lacks the typical zincribbon motif present in most ribosomal proteins. Furthermore, several EMSAs confirmed that the purified AdcR protein binds specifically to the promoters of both genes (Fig. 1).

The other two ORFs of strain P1/7 presenting an AdcRbinding sequence correspond to the S. suis 89/1591 homologues previously identified as ssuiDRAFT 1240 ORF $(a d c R)$ and ssuiDRAFT 0103 ORF (encoding a putative zinc-binding streptococcal lipoprotein) (Aranda et al., 2008). These ORFs were annotated as ssu0112 and ssu0308, respectively, in the $S$. suis $\mathrm{P} 1 / 7$ strain (Table 1 ). As in strain 89/1591, in P1/7 adcR is the first gene of a transcriptional unit, which also contains three components of an ABC transporter (Ssu0113-0115) involved in zinc/ manganese uptake (Table 1). Furthermore, it is worth noting that both $a d c R$-regulated ssuiDRAFT 0103 and 1237 lipoproteins (Ssu0308 and Ssu0115 P1/7 homologues) have been reported to be immunogenic and able to confer protection against S. suis in mice (Aranda et al., 2008). In this regard, it is known that lipoproteins are the $A B C$ transporter components most exposed outside the Grampositive cell surface (Hantke, 2001; Sutcliffe \& Russell, 1995). Taken together, these data suggest that $a d c R$ mutant cell surface-associated proteins could be candidates for effective vaccines against $S$. suis infection. (a)
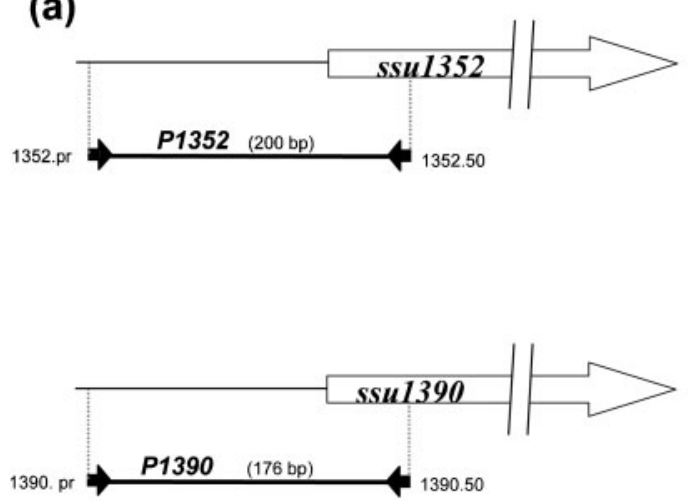

(b)

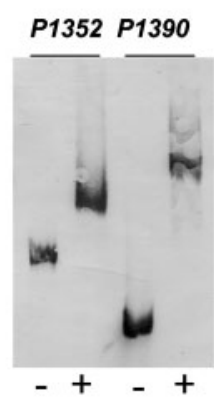

Fig. 1. (a) Schematic representation of ssu1352 and ssu1390 ORFs with their promoter regions (P1352 and $\mathrm{P} 1390$, respectively). Small arrows indicate the primer pairs used to amplify the promoters for EMSA experiments. (b) Electrophoretic mobility of the DNA fragments containing the indicated $S$. suis promoters in the absence $(-)$ or presence $(+)$ of purified S. suis AdcR protein. 
Table 1. Nomenclature of adcR-regulated genes in $P 1 / 7$ and $89 / 1591$ S. suis strains

\begin{tabular}{|c|c|c|c|}
\hline ORF $(\mathrm{P} 1 / 7)^{\star}$ & ORF $(89 / 1591) \dagger$ & Putative function & $\begin{array}{l}\text { AdcR-binding sequence } \\
\text { and position } \ddagger\end{array}$ \\
\hline $\operatorname{ssu} 0112 \S$ & ssuiDRAFT 1240 & Regulatory protein (AdcR) & TTAACAAGTTAA $(-28)$ \\
\hline ssu0114§ & ssuiDRAFT 1238 & $\mathrm{ABC}$ transporter component; permease & \\
\hline ssu0115\$ & ssuiDRAFT 1237 & ABC transporter component; zinc-binding lipoprotein & \\
\hline ssu0308॥ & ssuiDRAFT 0103 & $\mathrm{ABC}$ transporter component; zinc-binding lipoprotein & TTAACTGGTTAA $(-26)$ \\
\hline ssu 1390 & ssuiDRAFT 0326 & Internalin A & TTAACTGGTTAA $(-31)$ \\
\hline ssu1352 & ssuiDRAFT 0240 & 30 S ribosomal protein $\mathrm{S} 14$ & TTAACTGGTTAA $(-36)$ \\
\hline ssu1103 & ssuiDRAFT 1606 & Pht protein & TTAACTAGTAAA $(-230)$ \\
\hline
\end{tabular}

*ORF name in S. suis strain P1/7 (http://www.sanger.ac.uk/Projects/S_suis/).

†ORF name in S. suis strain 89/1591 (http://www.ncbi.nlm.nih.gov).

$\$$ Position with respect to its predicted translational starting point.

$\$ / I$ Genes constituting a single transcriptional unit. The AdcR motif is located upstream of the first gene listed.

In order to evaluate the role of the AdcR protein in the regulation of genes whose products could present immunogenic and/or protective abilities, the construction and characterization of an $a d c R$ in-frame deletion mutant was carried out as described in Methods. After electrotransformation with plasmid pSET4 $\Delta \mathrm{adcR}$ to replace the wild-type $a d c R$ gene of $S$. suis strain P1/7 with an $a d c R$ deleted gene, the $S$. suis UA5000 strain $\left(\mathrm{AdcR}^{-}\right)$was obtained, with the deletion confirmed by both PCR and sequencing (data not shown). The repressor function of the AdcR protein upon these genes was confirmed by the fact that the null- $a d c R$ strain showed derepression of all AdcRregulated ORFs (Fig. 2). Moreover, none of the non- $a d c R$ regulated ssu0626, ssu1246 and 0118 genes (lacking an AdcR-binding site in their promoter regions) was induced in the $a d c R$ mutant strain when compared with the wildtype strain (results not shown).

\section{The AdcR protein regulates an S. suis gene that encodes a ribosomal protein paralogue}

It has been proposed that genes that encode ribosomal protein paralogues are regulated by $\mathrm{AdcR}$ and thus contribute to survival during zinc starvation (Panina et al., 2003). This hypothesis is based on the fact that original copies of ribosome proteins present zinc-ribbon motifs (C$\left.\mathrm{X}_{2}-\mathrm{C}-\mathrm{X}_{\mathrm{n}}-\mathrm{C}-\mathrm{X}_{2}-\mathrm{C}\right)$ and are thus likely to bind zinc, while paralogous copies lack these motifs. However, the presence of zinc in the ribosomes seems to be required to stabilize them at high temperature only in thermophilic bacteria and Archaea (Makarova et al., 2001). Therefore, it has been suggested that under conditions of zinc starvation, the induction of paralogous genes could lead to the incorporation of these paralogous proteins into the ribosomes in place of the original ribosomal proteins. After degradation of the original zinc-containing ribosomal proteins, the metal becomes available to be used by other zinc-

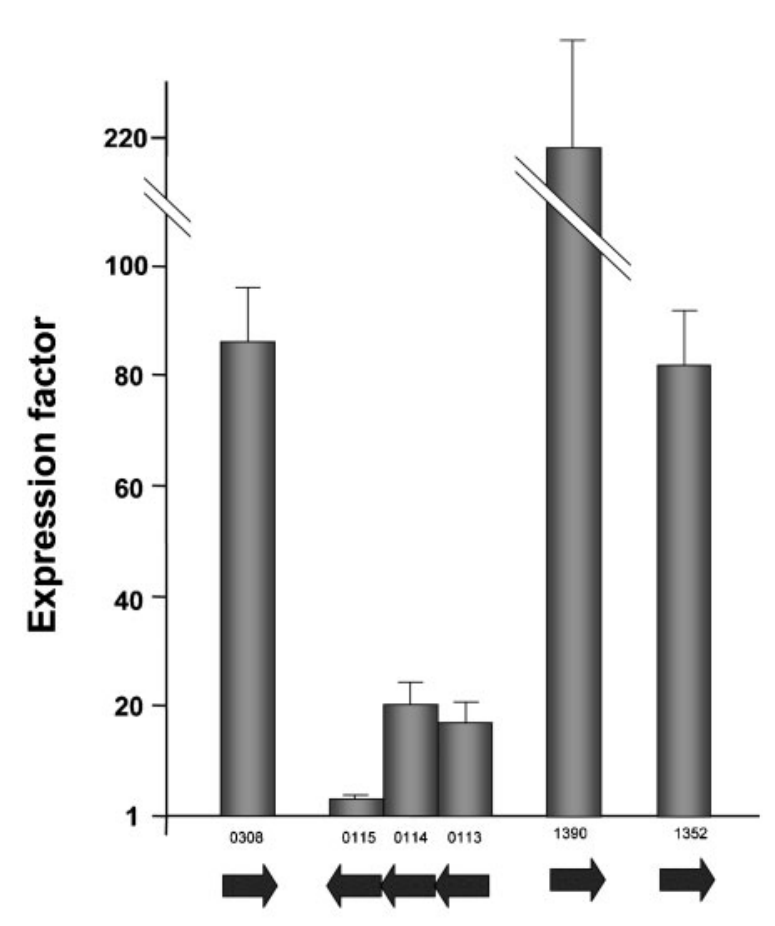

AdcR-regulated genes

Fig. 2. Expression factors of several ORFs (Table 1 ) in the $S$. suis adcR mutant (UA5000). The expression factor is the ratio of the mRNA concentration of each gene from the UA5000 strain with respect to the wild-type strain (P1/7). The amount of mRNA of each gene was determined by using a standard curve generated by the amplification of an internal fragment of the $S$. suis P1/7 ORF encoding cysteine synthase (see Supplementary Table S2 for primer sequences). The results are the mean of two independent experiments, each carried out in duplicate. Error bars, SD. 
dependent proteins. Scenarios in concordance with this hypothesis have been reported during zinc starvation for ribosomal proteins of B. subtilis and Streptococcus pyogenes (Brenot et al., 2007; Makarova et al., 2001). In accordance with this, our results showed that expression of ssu1352 ORF in the $a d c R$-knockout strain, encoding ribosomal protein S14, is approximately 100 -fold higher than in the wild-type strain (Fig. 2). In this respect, an ORF (ssu0083) encoding the putative original $30 \mathrm{~S}$ ribosomal protein S14 ORF is also present in the complete genome of S. suis strain P1/7. As expected, the predicted product of the ssu0083 ORF includes a zinc-ribbon motif (CEKCGRPHSVYRKFKLCRVC) which is absent in the AdcR-regulated ssu1352 ORF (data not shown). Moreover, expression of the ssu0083 ORF, which lacks the AdcR-binding site in its promoter region, is not increased in the $a d c R$ mutant strain (data not shown). Although further experiments are needed to fully evaluate its validity, our data support the hypothesis that genes encoding ribosomal protein paralogues regulated by AdcR contribute to zinc homeostasis in streptococci.

\section{Two Pht proteins are under AdcR regulation in S. suis}

SDS-PAGE analysis of cell surface-associated proteins of both the $a d c R$ mutant and wild-type strains revealed the overexpression of two proteins of approximately 120 and $100 \mathrm{kDa}$ in the adcR-knockout strain (Fig. 3). To identify them, both protein gel bands were in-gel digested with trypsin and the peptides obtained were identified by MALDI-TOF MS (data not shown). The results indicated that the two proteins corresponded to ssu0309 and ssu1 103 ORF products, which have both been annotated as Pht (pneumococcal histidine triad) proteins, showing scores of

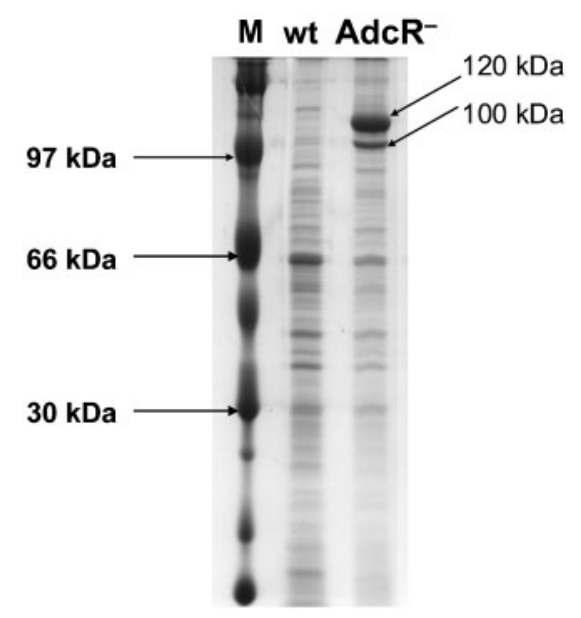

Fig. 3. SDS-PAGE profile of cell wall-associated proteins of both S. suis P1/7 (wt) and UA5000 (AdcR ${ }^{-}$) strains. Two overexpressed proteins of UA5000 cell extracts are indicated by arrows with their apparent molecular masses. The molecular mass marker (M) and its values are shown on the left of the figure.
165 and 73, respectively (using the MASCOT search engine, version 2.1). The predicted products of these two genes are proteins of 116.5 and $95.4 \mathrm{kDa}$ molecular mass, respectively, in agreement with the apparent molecular mass seen on SDS-PAGE (Fig. 3). Pht proteins have only been found in pathogenic streptococcal species and are involved in the invasion process (Adamou et al., 2001; Panina et al., 2003). This family of proteins shows a conserved and repeated motif $\mathrm{HxxHxH}$ (histidine triad motif) which is implicated in zinc binding (Adamou et al., 2001; Panina et al., 2003; Riboldi-Tunnicliffe et al., 2005).

Overexpression of Ssu1103 and Ssu0309 proteins in the adcR mutant strain suggested that they are under the control of AdcR. In this respect, it must be noted that an AdcR-like motif (TTAACTAGTAAA) is found at position -230 with respect to the ssu1103 predicted translational starting point. For this reason, the ability of $S$. suis AdcR protein to bind the promoter region of ssu1103 ORF was analysed by EMSA. Data showed that the AdcR protein specifically binds to the promoter of this gene (Fig. 4).

Moreover, and since ssu0308 and ssu0309 ORFs were demonstrated to be co-transcribed by RT-PCR analysis (data not shown), a search for putative AdcR boxes upstream of this transcriptional unit was carried out. As expected, one motif with these characteristics (TTAACTGGTTAA) was found at position -26 with respect to the predicted translational starting point of the ssu0308 ORF, whose product is homologous (99\% identity) to the protein encoded by the AdcR-regulated ssuiDRAFT 0103 ORF of the $89 / 1591$ S. suis strain (Table 1). Furthermore, EMSA experiments demonstrated that the S. suis AdcR protein is able to bind this AdcR-like sequence present upstream of the ssu0308-ssu0309 transcriptional unit (data not shown). In accordance with all these data, derepression of both ssu1103 and ssu0309 ORFs was observed in the adcR mutant strain (Fig. 5).

Furthermore, Pht proteins are involved in the host-cellpathogen interaction, and it has been hypothesized that they have evolved to become sensitive to AdcR regulation in order to exploit zinc as a compartment-specific regulatory cue (Brenot et al., 2007). It is also noteworthy that Pht proteins present hydrophobic leader sequences, which presumably target these proteins to the bacterial cell surface and induce specific antibodies against pneumococcal sepsis, suggesting that $\mathrm{Pht}$ proteins could serve as effective vaccines against streptococcal infections (Adamou et al., 2001; Kunitomo et al., 2008). Our data effectively demonstrate the presence of Pht proteins at the S. suis cell surface and that AdcR protein is not only involved in cation homeostasis but also controls putative virulence factors.

\section{Protective capacities of cell surface-associated proteins of adcR, fur and adcR fur S. suis mutants}

As mentioned above, the adcR mutant strain overexpresses genes encoding predicted immunogenic proteins. For that 
(a)

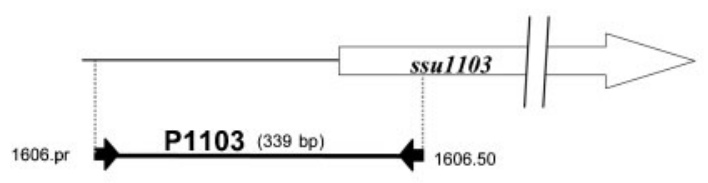

(b)

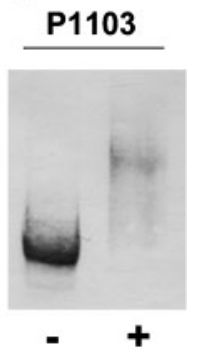

Fig. 4. (a) Schematic representation of the ssu1103 ORF with its promoter region (P1103). Small arrows indicate the primer pairs used to amplify the promoters for EMSA experiments. (b) Electrophoretic mobility of the DNA fragment containing the indicated $S$. suis promoter in the absence (-) or presence $(+)$ of purified $S$. suis AdcR protein. reason, the cell surface-associated proteins of both wildtype and $a d c R$-null strains were analysed on Western blots by using immunologically reactive serum obtained from surviving mice after inoculation with the S. suis $89 / 1591$ wild-type strain. Results showed that the adcR mutant strain extracts presented more immunological proteins than the wild-type strain (Fig. 6). However, no significant protective effect was obtained when the cell surfaceassociated proteins of the adcR mutant strain were inoculated into mice prior to a challenge with the wildtype $S$. suis $89 / 1591$ strain (data not shown). This result suggested that adcR-regulated proteins associated with the cell surface are not sufficient to prevent the infective process in mice. This could be due to the amount of immunogenic proteins not being sufficient or their exposure to the external medium not being as extensive

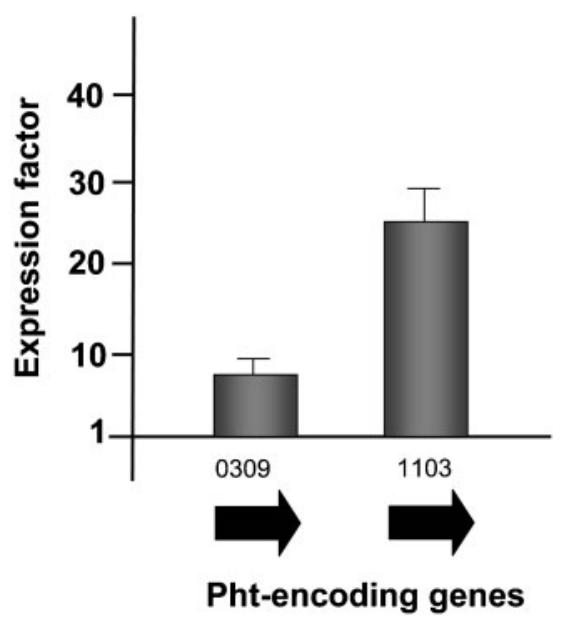

Fig. 5. Expression factors of Pht-encoding ssu0309 and ssu1103 ORFs in the $S$. suis adcR mutant (UA5000). The expression factor is the ratio of the mRNA concentration of each gene from the UA5000 strain with respect to the wild-type strain (P1/7). The amount of mRNA of each gene was determined by using a standard curve generated by the amplification of an internal fragment of the $S$. suis P1/7 ORF encoding cysteine synthase (see Supplementary Table S2 for primer sequences). The results are the means of two independent experiments, each carried out in duplicate. Error bars, SD. as necessary to confer good protection. In fact, previous data have demonstrated that a recombinant adcR-regulated protein (SsuiDRAFT 0103) is sufficient to confer significant protection against this pathogen in mice (Aranda et al., 2008).

A search in the NCBI database (http://www.ncbi.nlm.nih. gov/) allowed the identification of the Streptococcus thermophilus fur gene (gene ID: 3166852). Then, this gene was used as a probe against the Sanger BLAST server (http:// www.sanger.ac.uk/Projects/S_suis/), enabling the identification of the S. suis P1/7 fur gene, which has a high degree of identity (64\%) to the S. thermophilus fur gene. As mentioned above, the Fur protein binds iron in the cytoplasm of many bacterial cells and blocks the transcription of iron-regulated genes, several of which express strongly immunogenic proteins (Escolar et al., 1999; Hantke, 1981; Ratledge \& Dover, 2000). After electrotransformation with plasmid pSET4 $\Delta$ fur to replace the wild-type fur gene of $S$. suis P1/7 strain with a fur-deleted gene, a fur-defective $S$. suis strain (UA5001) was obtained, with the deletion confirmed by both PCR and sequencing (data not shown). However, no significant protective effect was obtained when the cell surface-associated proteins of the fur mutant strain were used to inoculate mice prior to a challenge with the wild-type S. suis 89/1591 strain (data not shown). Then, to increase the putative immunogenic capacity of both $S$. suis mutants, the construction of a double mutant strain (UA5002, AdcR ${ }^{-}$Fur $^{-}$) was carried out. The presence of both the $a d c R$ and fur gene deletions was confirmed by both PCR and sequencing (data not shown).

Analysis of the SDS-PAGE profile of S. suis UA5002 $\left(\right.$ AdcR $^{-}$Fur $^{-}$) cell surface-associated proteins revealed that the double mutant strain presented a higher number of immunogenic proteins than both $S$. suis single mutants (Fig. 6). In order to determine whether the induction of this immunogenic response was enough to protect mice against $S$. suis infection, three groups of eight mice were immunized twice with $50 \mu \mathrm{g}$ cell surface-associated proteins of the double mutant strain, $50 \mu \mathrm{g}$ cell surfaceassociated proteins of the wild-type strain, or the solvent including the adjuvant (as a control). When the animals were challenged with $10^{8}$ c.f.u. S. suis $89 / 1591$, all the mice belonging to either the control group or the group 
(a)

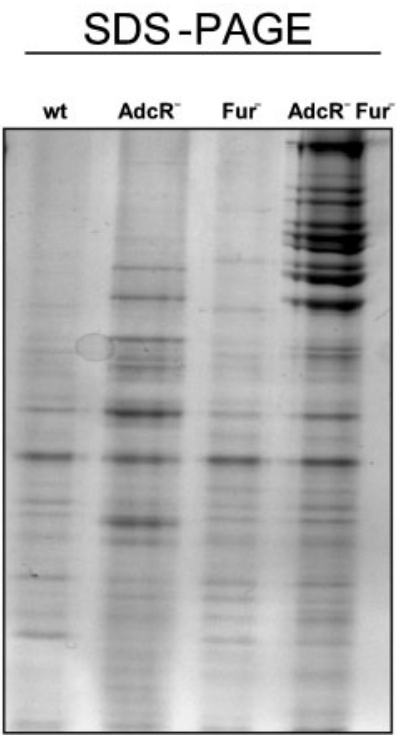

(b) WESTERN

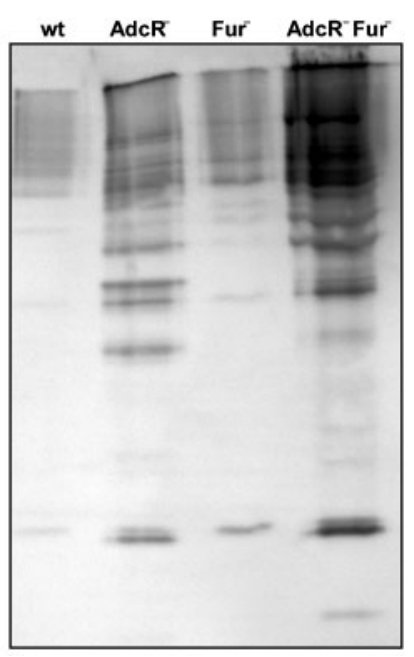

Fig. 6. (a) SDS-PAGE profile of $S$. suis $P 1 / 7$ wild-type (wt), UA5000 (AdcR ${ }^{-}$), UA5001 (Fur ${ }^{-}$) and UA5002 (AdcR ${ }^{-}$Fur $^{-}$) strains. (b) Western blot analysis with serum recovered from mice infected with $S$. suis strain 89/1591. The analysis was carried out with cell surfaceassociated proteins obtained from S. suis $\mathrm{P} 1 / 7$ wild-type (wt), UA5000 (AdcR $\left.{ }^{-}\right)$, UA5001 (Fur $^{-}$) and UA5002 (AdcR ${ }^{-}$Fur $^{-}$) strains. vaccinated with the wild type extract died within 5 days of infection (Fig. 7). In contrast, $50 \%$ of mice in the group vaccinated with the double mutant extract inoculated with an identical dose of the virulent strain remained alive throughout the experiment (Fig. 7). In addition, both wildtype and adcR fur double mutant cell surface-associated proteins were analysed on Western blots by using immunologically reactive serum obtained from mice surviving after inoculation with the S. suis 89/1591 wild-

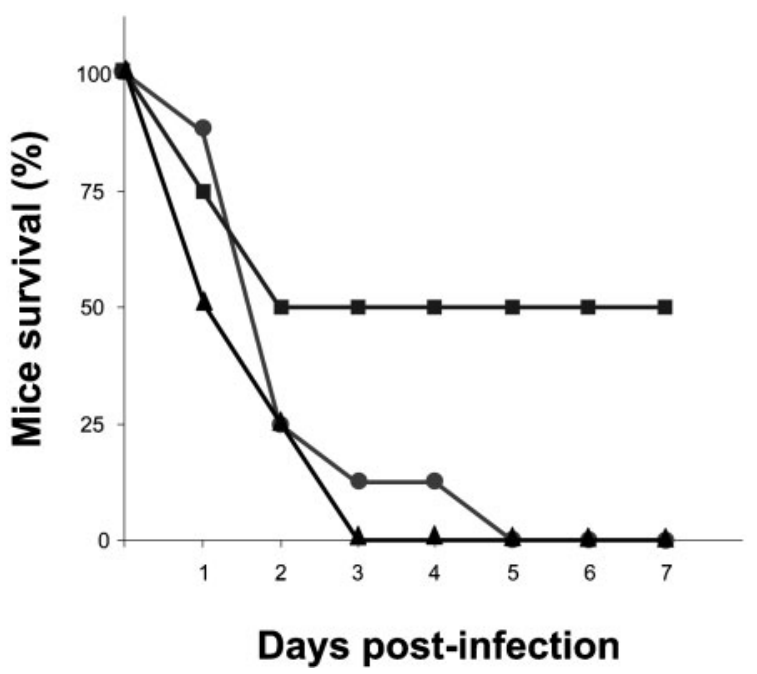

Fig. 7. Survival curve of mice vaccinated with cell surfaceassociated proteins of wild-type P1/7 strain ( $)$, cell surfaceassociated proteins of adcR fur UA5002 strain (ם), or solvent including the adjuvant as a negative control $(\boldsymbol{\Lambda})$. After the last immunization, an intraperitoneal challenge with $10^{8}$ c.f.u. wild-type S. suis $89 / 1591$ cells was administered to each animal ( $n=8$ mice per group). type strain. The results provided clear evidence that the protective effect of the double mutant extract was correlated with specific antibodies against the overexpressed antigens of this strain (Fig. 6). It is worth noting that negative results (no reactivity) were obtained when all the $S$. suis extracts used in this work were tested with serum from non-infected mice (data not shown). All these data indicated that the combination of cell surface-associated proteins under the control of either zinc/manganese- or iron-uptake regulators (AdcR and Fur, respectively) not only is immunogenic but also confers a statistically significant level of protection $(P=0.038)$ against $S$. suis challenge to mice immunized with these proteins.

To sum up, the data reported in this work demonstrate that the use of cell surface extracts of $S$. suis double mutants defective in genes regulating either $\mathrm{Zn}^{2+} / \mathrm{Mn}^{2+}$ or $\mathrm{Fe}^{2+}$ cations is able to protect mice against challenge with $S$. suis. This double mutant-based strategy has not to our knowledge been used to date and can be applied to other virulent bacterial species. Thus, mutagenesis of this kind of regulator makes it possible to obtain effects in the overexpression of divalent cation-uptake transporters on the bacterial cell surface similar to those induced by chelating agents, overcoming problems associated with the use of these chemicals, such as poor bacterial cell growth, and simplifying the bulk production of this antigen source. In addition, the data reported in this work indicate that the use of purified immunogenic proteins such as Pht or SsuiDRAFT 0103 is a more promising vaccination strategy than the use of protein extracts.

\section{ACKNOWLEDGEMENTS}

We are very grateful to Dr T. Sekizaki, National Institute of Animal Health, Ibaraki, Japan, for providing the pSET4s plasmid used in this work. We thank Joan Ruiz and Sonia Lacouture for their excellent 
technical assistance. This work was funded by grants AGL2005-03574 from the Ministerio de Educación y Ciencia (MEC) de España and 2005SGR-533 from the Departament d'Universitats, Recerca i Societat de la Informació (DURSI) de la Generalitat de Catalunya. J.A. is the recipient of a pre-doctoral fellowship from the Universitat Autònoma de Barcelona. N.F. is the recipient of a Natural Sciences and Engineering Research Council of Canada postgraduate scholarship.

\section{REFERENCES}

Abella, M., Rodriguez, S., Paytubi, S., Campoy, S., White, M. F. \& Barbé, J. (2007). The Sulfolobus solfataricus radA paralogue sso0777 is DNA damage inducible and positively regulated by the Stal protein. Nucleic Acids Res 35, 6788-6797.

Adamou, J. E., Heinrichs, J. H., Erwin, A. L., Walsh, W., Gayle, T., Dormitzer, M., Dagan, R., Brewah, Y. A., Barren, P. \& other authors (2001). Identification and characterization of a novel family of pneumococcal proteins that are protective against sepsis. Infect Immun 69, 949-958.

Andrews, S. C., Robinson, A. K. \& Rodriguez-Quinones, F. (2003). Bacterial iron homeostasis. FEMS Microbiol Rev 27, 215-237.

Aranda, J., Garrido, M. E., Cortes, P., Llagostera, M. \& Barbé, J. (2008). Analysis of the protective capacity of three Streptococcus suis proteins induced under divalent-cation-limited conditions. Infect Immun 76, 1590-1598.

Babich, H. \& Stotzky, G. (1978). Toxicity of zinc to fungi, bacteria, and coliphages: influence of chloride ions. Appl Environ Microbiol 36, 906-914.

Brenot, A., Weston, B. F. \& Caparon, M. G. (2007). A PerR-regulated metal transporter (PmtA) is an interface between oxidative stress and metal homeostasis in Streptococcus pyogenes. Mol Microbiol 63, 11851196.

Bsat, N. \& Helmann, J. D. (1999). Interaction of Bacillus subtilis Fur (ferric uptake repressor) with the $d h b$ operator in vitro and in vivo. J Bacteriol 181, 4299-4307.

Campoy, S., Mazon, G., Fernandez de Henestrosa, A. R., Llagostera, M., Monteiro, P. B. \& Barbe, J. (2002). A new regulatory DNA motif of the gamma subclass Proteobacteria: identification of the LexA protein binding site of the plant pathogen Xylella fastidiosa. Microbiology 148, 3583-3597.

Chamnongpol, S., Dodson, W., Cromie, M. J., Harris, Z. L. \& Groisman, E. A. (2002). $\mathrm{Fe}(\mathrm{III})$-mediated cellular toxicity. $\mathrm{Mol}$ Microbiol 45, 711-719.

Escolar, L., Perez-Martin, J. \& de Lorenzo, V. (1999). Opening the iron box: transcriptional metalloregulation by the Fur protein. J Bacteriol 181, 6223-6229.

Fontaine, M. C., Perez-Casal, J. \& Willson, P. J. (2004). Investigation of a novel DNase of Streptococcus suis serotype 2. Infect Immun 72, 774-781.

Garmory, H. S. \& Titball, R. W. (2004). ATP-binding cassette transporters are targets for the development of antibacterial vaccines and therapies. Infect Immun 72, 6757-6763.

Garrido, M. E., Bosch, M., Bigas, A., Badiola, I., Barbé, J. \& Llagostera, M. (2008). Heterologous protective immunization elicited in mice by Pasteurella multocida fur ompH. Int Microbiol 11, 17-24.

Gottschalk, M. \& Segura, M. (2000). The pathogenesis of the meningitis caused by Streptococcus suis: the unresolved questions. Vet Microbiol 76, 259-272.

Hantke, K. (1981). Regulation of ferric iron transport in Escherichia coli K12: isolation of a constitutive mutant. Mol Gen Genet 182, 288-292.

Hantke, K. (2001). Bacterial zinc transporters and regulators. Biometals 14, 239-249.
Higgins, R., Gottschalk, M., Boudreau, M., Lebrun, A. \& Henrichsen, J. (1995). Description of six new capsular types (29-34) of Streptococcus suis. J Vet Diagn Invest 7, 405-406.

Kunitomo, E., Terao, Y., Okamoto, S., Rikimaru, T., Hamada, S. \& Kawabata, S. (2008). Molecular and biological characterization of histidine triad protein in group A streptococci. Microbes Infect 10, 414-423.

Laemmli, U. K. (1970). Cleavage of structural proteins during the assembly of the head of bacteriophage T4. Nature 227, 680-685.

Loo, C. Y., Mitrakul, K., Voss, I. B., Hughes, C. V. \& Ganeshkumar, N. (2003). Involvement of the adc operon and manganese homeostasis in Streptococcus gordonii biofilm formation. J Bacteriol 185, 2887-2900.

Makarova, K. S., Ponomarev, V. A. \& Koonin, E. V. (2001). Two C or not two C: recurrent disruption of Zn-ribbons, gene duplication, lineage-specific gene loss, and horizontal gene transfer in evolution of bacterial ribosomal proteins. Genome Biol 2, RESEARCH 0033.

Miller, J. H. (1992). A Short Course in Bacterial Genetics. Cold Spring Harbor, NY: Cold Spring Harbor Laboratory.

Panina, E. M., Mironov, A. A. \& Gelfand, M. S. (2003). Comparative genomics of bacterial zinc regulons: enhanced ion transport, pathogenesis, and rearrangement of ribosomal proteins. Proc Natl Acad Sci U S A 100, 9912-9917.

Patzer, S. I. \& Hantke, K. (2000). The zinc-responsive regulator Zur and its control of the $z n u$ gene cluster encoding the ZnuABC zinc uptake system in Escherichia coli. J Biol Chem 275, 24321-24332.

Ratledge, C. \& Dover, L. G. (2000). Iron metabolism in pathogenic bacteria. Annu Rev Microbiol 54, 881-941.

Riboldi-Tunnicliffe, A., Isaacs, N. W. \& Mitchell, T. J. (2005). $1.2 \AA$ crystal structure of the $S$. pneumoniae PhtA histidine triad domain a novel zinc binding fold. FEBS Lett 579, 5353-5360.

Sambrook, J. \& Russell, D. W. (2001). Molecular Cloning: a Laboratory Manual, 3rd edn. Cold Spring Harbor, NY: Cold Spring Harbor Laboratory.

Staats, J. J., Feder, I., Okwumabua, O. \& Chengappa, M. M. (1997). Streptococcus suis: past and present. Vet Res Commun 21, 381-407.

Sutcliffe, I. C. \& Russell, R. R. (1995). Lipoproteins of Gram-positive bacteria. J Bacteriol 177, 1123-1128.

Takamatsu, D., Osaki, M. \& Sekizaki, T. (2001a). Construction and characterization of Streptococcus suis-Escherichia coli shuttle cloning vectors. Plasmid 45, 101-113.

Takamatsu, D., Osaki, M. \& Sekizaki, T. (2001b). Thermosensitive suicide vectors for gene replacement in Streptococcus suis. Plasmid 46, 140-148.

Tanabe, M., Atkins, H. S., Harland, D. N., Elvin, S. J., Stagg, A. J., Mirza, O., Titball, R. W., Byrne, B. \& Brown, K. A. (2006). The ABC transporter protein OppA provides protection against experimental Yersinia pestis infection. Infect Immun 74, 3687-3691.

Tavares, F. \& Sellstedt, A. (2000). A simple, rapid and nondestructive procedure to extract cell wall-associated proteins from Frankia. J Microbiol Methods 39, 171-178.

Touati, D. (2000). Iron and oxidative stress in bacteria. Arch Biochem Biophys 373, 1-6.

Wollert, T., Heinz, D. W. \& Schubert, W. D. (2007). Thermodynamically reengineering the listerial invasion complex InlA/E-cadherin. Proc Natl Acad Sci U S A 104, 13960-13965.

Yu, H., Jing, H., Chen, Z., Zheng, H., Zhu, X., Wang, H., Wang, S., Liu, L., Zu, R. \& other authors (2006). Human Streptococcus suis outbreak, Sichuan, China. Emerg Infect Dis 12, 914-920.

Edited by: T. J. Mitchell 